\title{
Integrated organic light-emitting device/fluorescence-based chemical sensors
}

\author{
V. Savvate'eva) \\ Ames Laboratory-USDOE and Department of Physics, Iowa State University, Ames, Iowa 50011 \\ Z. Chen-Esterlit and J. W. Aylott ${ }^{\text {b) }}$ \\ Department of Chemistry, University of Michigan, Ann Arbor, Michigan 48109 \\ B. Choudhury, C.-H. Kim, L. Zou, J. H. Friedl, R. Shinar, and J. Shinarc) \\ University, Ames, Iowa 50011 \\ R. Kopelman ${ }^{\text {d) }}$ \\ Department of Chemistry, University of Michigan, Ann Arbor, Michigan 48109
}

Ames Laboratory-USDOE, Department of Physics, and Microelectronics Research Center, Iowa State

(Received 9 April 2002; accepted 10 September 2002)

\begin{abstract}
A fluorescent chemical sensor platform, integrating an organic light-emitting device (OLED) light-source with a fluorescent probe, is demonstrated for a subsecond-fast oxygen sensor. The integration results in strong light coupling and negligible heating of the sensor film or analyte. The potential in vivo operation of compact, stand-alone, battery-powered, OLED-based miniaturized sensor arrays for chemical and biological applications is discussed. (c) 2002 American Institute of Physics. [DOI: 10.1063/1.1518154]
\end{abstract}

The structural integration of a fluorescent sensor film with its light source is highly desirable for the development of miniaturized sensor arrays. It would address the need for miniaturized devices in medical and environmental testing, high-throughput drug discovery, and detection of inorganic gases, volatile organic compounds, pathogens, warfare agents, and in vivo biological compounds, and organisms. In general, fluorescence-based (bio)chemical sensors require three components: A fluorescing sensing element (the fluorescence of which probes the analyte), a light source that excites the sensing element, and a photodetector. Current light sources such as lasers or inorganic light-emitting diodes either cannot be integrated with the other components due to size, geometrical, or operational constraints, or they involve intricate integration procedures. ${ }^{1}$ In addition, the heat generated can damage the sensor or analyte. This letter describes an integrated fluorescence-based oxygen sensor, which combines a fluorescent dye and an organic light-emitting device (OLED) in a very simple design. As shown below, such optimized sensors result in negligible heating of the sensor film or analyte, and could lead to applications in medical, (bio-) chemical, and environmental diagnostics at a molecular level, where microarray technology is required for highthroughput analysis and imaging. ${ }^{2}$

OLEDs have dramatically improved over the past decade, ${ }^{3}$ and commercial products incorporating them have begun to emerge. ${ }^{4}$ They are inherently advantageous as

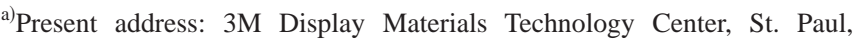
MN 55144.

b) Present address: Department of Chemistry, University of Hull, Hull HU6 7RX, UK.

c) Author to whom correspondence should be addressed; electronic mail shinar@ameslab.gov

d) Author to whom correspondence should be addressed; electronic mail: kopelman@umich.edu
}

low-voltage, ${ }^{3}$ miniaturized, ${ }^{5}$ and flexible light sources; ${ }^{6}$ green OLEDs with an external quantum efficiency $\eta_{\text {ext }}$ $\approx 18 \%^{7}$ and blue OLEDs with $\eta_{\mathrm{ext}} \approx 5.7 \%^{8}$ have been reported.

The integration strategy described in this letter is based on depositing the sensor film, a fluorescent dye embedded in a matrix, on one side of a transparent substrate, and the OLED on the other side. Figure 1 shows an envisioned array of such sensors. The sensing film is fully exposed to the gaseous or liquid analyte, while the OLED is sealed from it.

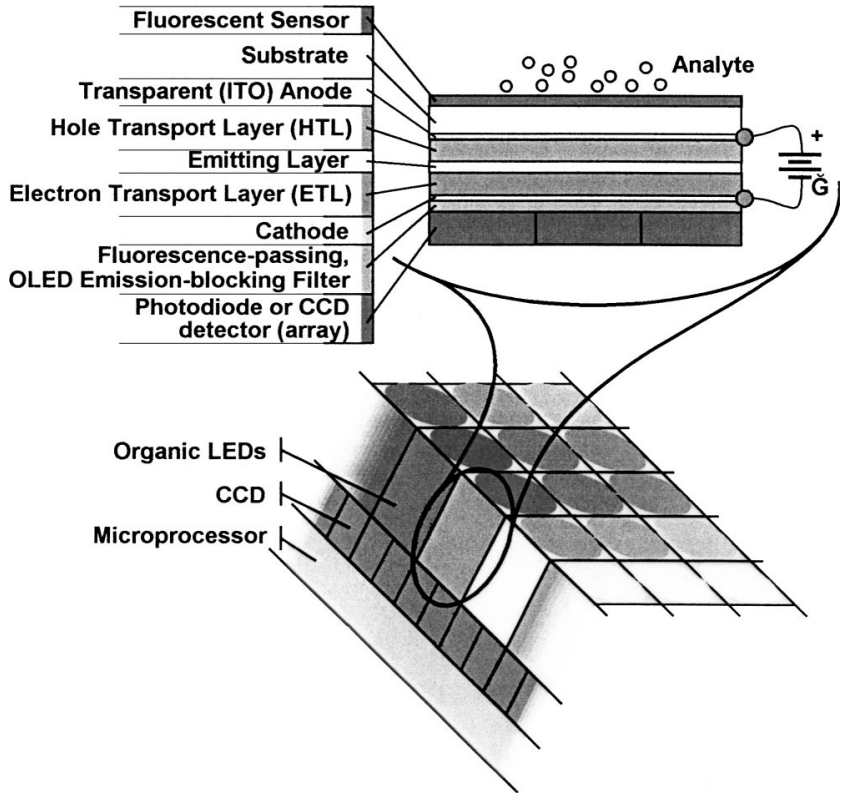

FIG. 1. Schematic of the proposed integrated fluorescence-based highdensity chemical sensor array. The detector can be a CCD or set of photodiodes that collect the light signal and transmit the resulting electrical signal to the processing unit. Zoom-in: Cross section of the integrated sensing device. 
In this geometry, the sensor film is on one side of the OLED array while the photodetector array is on the other side and monitors the fluorescence passing through the array. This "back-detection mode" is highly advantageous for integration in general, and in vivo operation in particular. It can be accomplished using either transparent OLEDs, ${ }^{9}$ or several OLED pixels in an array (where the fluorescence passing through the gaps between the pixels is monitored).

OLED arrays were fabricated by vacuum evaporation of the organic layers on indium tin oxide coated glass. They were based on blue-emitting $4,4^{\prime}$ - bis-(2,2' - diphenylvinyl)$1,1^{\prime}$-biphenyl (DPVBi) ${ }^{10,11}$ or blue-emitting perylene-doped 4,4' - bis(9-carbazolyl) biphenyl (Pe:CBP). ${ }^{12,13}$ The OLEDs were prepared as a matrix array of $\sim 1.5 \mathrm{~mm}$ diameter $\mathrm{Al}$ disk electrodes evaporated onto the organic layers. ${ }^{13}$

The oxygen sensor film was based on ruthenium tris(4,7diphenyl-1,10-phenanthroline) chloride [Ru(dpp)] dye ${ }^{14-16}$ $\mathrm{Ru}$ complexes are used as oxygen-sensitive dyes; the detection of oxygen is important for biomedical (e.g., glucose sensing ${ }^{17}$ ) and environmental applications. ${ }^{14-16} \mathrm{Ru}(\mathrm{dpp})$ is photostable and its strong photoluminescence (PL), with a $\sim 2 \mu$ s radiative lifetime, ${ }^{18}$ undergoes collisional quenching by molecular oxygen. ${ }^{19}$

The thin-film oxygen sensors were prepared by immobilizing $\mathrm{Ru}(\mathrm{dpp})$ in a sol-gel matrix. First, $\mathrm{Ru}(\mathrm{dpp})$ was blended with a sol, prepared from a methyltriethoxysilane precursor mixed with water and ethanol. The doped sol was spin coated onto cleaned, silanized glass slides and then aged at $70^{\circ} \mathrm{C}$. The resulting sensor films were optically transparent and crack free.

The measurements on the $\mathrm{Ru}(\mathrm{dpp})$ sensor films excited by integrated DPVBi-based OLEDs were carried out in air. The OLEDs were operated in a dc mode with a forward bias of $15-20 \mathrm{~V}$ or in a pulsed mode. The PL and electroluminescence (EL) spectra were measured in the "front-detection mode," where the sensing layer and the photodetector are on the same side of the OLED. The measurements on the oxygen sensors excited by the Pe:CBP OLEDs were performed in a gas flow cell that was alternately flooded with oxygen or argon; the sensor PL was monitored with either a Si photodiode or a photomultiplier tube (PMT).

Figure 2 presents the (1) PL, (2) PL excitation, and (3) absorption spectra of $\mathrm{Ru}(\mathrm{dpp})$ immobilized in the sol-gel matrix, and the normalized EL spectra of (4) blue DPVBi and (5) violet-blue Pe:CBP OLEDs. The dye PL peaks at 601 $\mathrm{nm}$. The EL strongly overlaps the Ru(dpp) PL excitation and absorption spectra, but there is little overlap between the EL and the sensor emission. To enhance the lifetime of OLEDs biased to high brightness and exposed to air, the DPVBi OLEDs were operated at a pulsed bias of 28-31 V, with a pulse width of 1-7 $\mu$ s and a repetition rate of $13-120 \mathrm{kHz}$. As expected, the pulsed and dc EL spectra were identical. ${ }^{10}$

The ability to operate the OLED in a pulsed mode allows us to explore its use in a PL lifetime-detection mode. This mode overcomes drawbacks of intensity-based measurements, such as errors due to dye bleaching and changes in excitation intensity, since quantifying the analyte via the PL lifetime does not require calibration. Moreover, detrimental sensor or analyte heating can be easily eliminated by reducing the duty cycle (see below). Figure 3 shows an applied

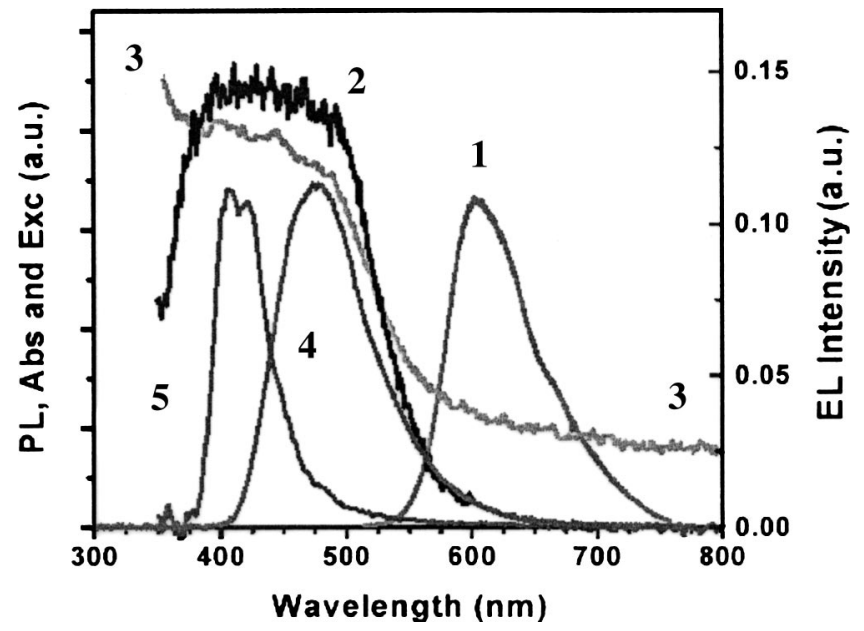

FIG. 2. (1) PL, (2) PL excitation, and (3) absorption spectra of the Ru(dpp) dye immobilized within a sol-gel matrix, measured in air. (4) The EL of blue DPVBi and (5) the EL of CBP OLEDs.

rectangular bias pulse, the PL decay, and the blue OLED emission collected through a band-pass filter. The inset shows the EL decay and the applied pulse. The sensor PL decays with the expected lifetime of $\sim 2 \mu \mathrm{s} .{ }^{20}$

The oxygen sensing measurements were performed using Pe:CBP OLEDs. Figure 4 shows the subsecond response of the sensor to alternate $7 \mathrm{psi} \mathrm{Ar}$ and $\mathrm{O}_{2}$ flows. The intensity is quenched by $72 \%$; the sensor response indicates an uncertainty of ca. $\pm 1 \%$ in the oxygen concentration.

The acute temperature sensitivity of chemical and biological sensors, as well as analytes, hampered the development of working fluorescence-based integrated arrays. The results shown in Fig. 4 enable us to address this issue. They were obtained at $V=16 \mathrm{~V}$ and $I=30 \mu \mathrm{A}$ (current density $J \approx 1.7 \mathrm{~mA} / \mathrm{cm}^{2}$ ), i.e., they produced a Joule power of $\sim 0.5 \mathrm{~mW}$. This power is easily dissipated by the glass substrate with negligible heating of the sensor film or analyte. At

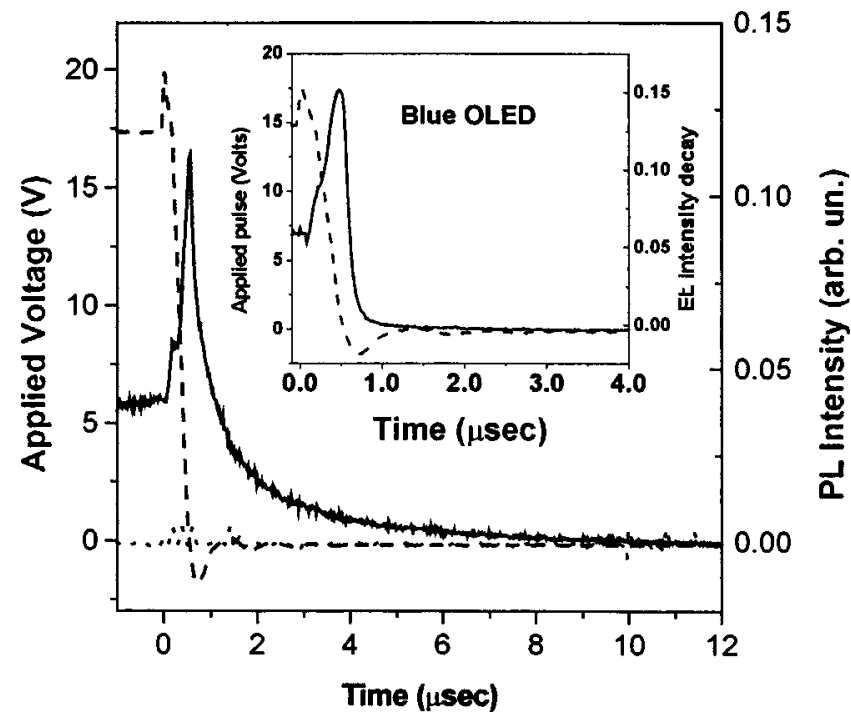

FIG. 3. Time-resolved PL of the oxygen sensor (solid line) and blue EL of the DPVBi OLED (dotted line), both obtained through a $15 \mathrm{~nm}$ band-pass filter at $600 \mathrm{~nm}$, from a $4.6 \mu \mathrm{s}$ bias pulse (dashed line) with a repetition rate $\nu_{c}=51.4 \mathrm{kHz}$. Inset: Time-resolved EL of the DPVBi blue OLED (solid line) generated by the bias pulse (dotted line); $\nu_{c}=47 \mathrm{kHz}$ 

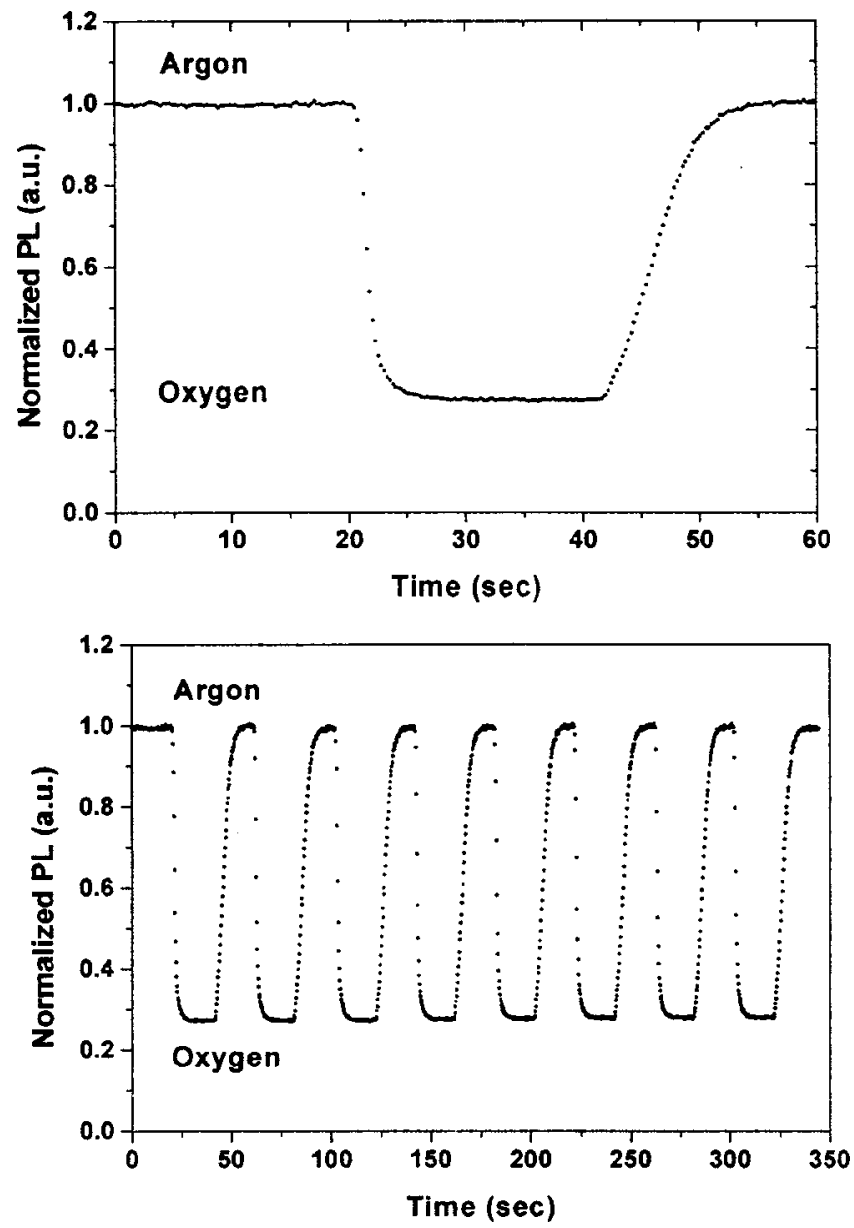

FIG. 4. $\mathrm{Ru}(\mathrm{dpp})$ sensor response to $\mathrm{Ar}$ and $\mathrm{O}_{2}$ vs time. The $\mathrm{Ru}(\mathrm{dpp})$ was excited by a blue Pe:CBP OLED, operated in a dc mode at $16 \mathrm{~V}$; the injected current was $I=50 \mu \mathrm{A}$, and the brightness was $\sim 1200 \mathrm{Cd} / \mathrm{m}^{2}$. The PL of the $\mathrm{Ru}(\mathrm{dpp})$ saturated the Hamamatsu R928 PMT. The gas flow was 7 psi for both $\mathrm{Ar}$ and $\mathrm{O}_{2}$.

the same time, it yielded an OLED brightness of $1200 \mathrm{Cd} / \mathrm{m}^{2}$, implying $\eta_{\mathrm{ext}} \approx 1.1 \%$ and a power efficiency $\approx 0.7 \%$. At this brightness, the $\mathrm{Ru}(\mathrm{dpp}) \mathrm{PL}$ saturated the PMT response. Hence, recently described blue OLEDs with $\eta_{\mathrm{ext}} \approx 5.7 \%^{8}$ would produce a Joule heat of only $\sim 0.1 \mathrm{~mW}$.

Pulsed mode operation also reduces the generated heat. For example, a $10 \mu \mathrm{s} 25 \mathrm{~V}(1 \mathrm{~mA})$ pulse, applied at $1 \mathrm{kHz}$, yields an average Joule power of only $0.25 \mathrm{~mW}$.

Note that the generated Joule heating is negligible despite the relatively low OLEDs' efficiency due to their nearideal coupling, i.e., due to their structural integration.

The foregoing oxygen sensor may be used as a basis for other sensors as well. For example, we have recently demonstrated an $\mathrm{OLED} / \mathrm{Ru}(\mathrm{dpp}) /$ glucose oxidase-based glucose sensor. $^{21}$

In summary, the integrated devices capitalize on the strengths of both OLED and miniaturized sensor technologies, and the near-ideal coupling between them. Future fabrication of multisensor microarrays for various analytes should be achievable by utilizing OLED pixels that emit at different bands. Because the sensing layer and the OLED are deposited in close proximity on opposite sides of a common substrate, the OLED and sensor pixel size can be minimal, while still yielding measurable PL, creating a highinformatioin density optoelectronic "nose." Indeed, recent developments in OLEDs confirm the feasibility of such multisensor microarrays. ${ }^{5,22}$ Therefore, this method should provide an ideal compact excitation source for incorporation into microfluidic systems, biochip-based devices, and highthroughput screening technologies, impacting the field of combinatorial biochemical sensors.

Ames Laboratory is operated by Iowa State University (ISU) for the United States Department of Energy under Contract No. W-7405-ENG-82. The work at ISU was supported by the Director for Energy Research, Office of Basic Energy Sciences, and by the ISU Research Foundation. The work at the University of Michigan was supported by National Science Foundation Grant No. DMR 9900434.

${ }^{1}$ E. J. Cho and F. V. Bright, Anal. Chem. 73, 3289 (2001).

${ }^{2}$ R. P. Ekins, Clin. Chem. 44, 2015 (1998), and references therein.

${ }^{3}$ R. H. Friend, R. W. Gymer, A. B. Holmes, J. H. Burroughes, R. N. Marks, C. Taliani, D. D. C. Bradley, D. A. Dos Santos, J. L. Brédas, M. Lögdlund, and W. R. Salaneck, Nature (London) 397, 121 (1999).

${ }^{4}$ www.pioneer.co.jp/press/release39.html (9/28/98); N. Bailey, Inf. Disp. 16(3), 12 (2000); S. Grossman, Electron. Design 48, 30 (2000).

${ }^{5}$ www.emagincorp.com

${ }^{6}$ G. Gustafsson, Y. Cao, G. M. Treacy, F. Klavetter, N. Colaneri, and A. J. Heeger, Nature (London) 357, 447 (1992); www.universaldisplay.com

${ }^{7}$ C. Adachi, M. A. Baldo, M. E. Thompson, and S. R. Forrest, J. Appl. Phys. 90, 5048 (2001).

${ }^{8}$ C. Adachi, R. C. Kwong, P. Djurovich, V. Adamovich, M. A. Baldo, M. E. Thompson, and S. R. Forrest, Appl. Phys. Lett. 79, 2082 (2001).

${ }^{9}$ G. Gu, V. Bulovic, P. E. Burrows, S. R. Forrest, and M. E. Thompson, Appl. Phys. Lett. 68, 2606 (1996).

${ }^{10}$ S. E. Shaheen, G. E. Jabbour, M. M. Morell, Y. Kawabe, B. Kippelen, N. Peyghambarian, M.-F. Nabor, R. Schlaf, E. A. Mash, and N. R. Armstrong, J. Appl. Phys. 84, 2324 (1998).

${ }^{11}$ V. Savvate'ev, J. H. Friedl, L. Zou, J. Shinar, K. Christensen, W. Oldham, L. J. Rothberg, Z. Chen-Esterlit, and R. Kopelman, Appl. Phys. Lett. 76, 1501 (2000)

${ }^{12}$ V. G. Kozlov, G. Parthasarathy, P. E. Burrows, S. R. Forrest, Y. You, and M. E. Thompson, Appl. Phys. Lett. 72, 144 (1998).

${ }^{13}$ L. Zou, V. Savvate'ev, J. Booher, C.-H. Kim, and J. Shinar, Appl. Phys. Lett. 79, 2282 (2001).

${ }^{14}$ W. L. Ramsey, J. M. Vanderkooi, and D. F. Wilson, Science 241, 1649 (1988).

${ }^{15}$ B. H. Weigl, A. Holobar, W. Trettnak, I. Klimant, H. Kraus, P. O'Leary, and O. Wolfbeis, J. Biotech. 32, 127 (1994).

${ }^{16}$ F. N. Castellano and J. R. Lakowicz, Photochem. Photobiol. 67, 179 (1998).

${ }^{17}$ Z. Rosenzweig and R. Kopelman, Anal. Chem. 68, 1408 (1996).

${ }^{18}$ E. R. Carraway, J. N. Demas, B. A. DeGraff, and J. R. Bacon, Anal. Chem. 63, 337 (1991).

${ }^{19}$ Fiber Optical Chemical Sensors and Biosensors, edited by O. S. Wolfbeis (CRC, Boca Raton, FL, 1991).

${ }^{20}$ S. Draxler, M. E. Lippitsch, I. Klimant, H. Kraus, and O. S. Wolfbeis, J. Phys. Chem. 99, 3162 (1995).

${ }^{21}$ A. Heller, Ann. Rev. Biomed. Eng. 1, 153 (1999).

${ }^{22}$ H. Kobayashi, S. Kanbe, S. Seki, H. Kigchi, M. Kimura, I. Yudasaka, S. Miyashita, T. Shimoda, C. R. Towns, J. H. Burroughes and R. H. Friend, Synth. Met. 111, 125 (2000); S.-C. Chang, J. Liu, J. Bharathan, Y. Yang, J. Onohara, and J. Kido, Adv. Mater. 11, 734 (1999); J. C. Sturm, F. Pschenitzka, T. R. Hebner, M. H. Lu, and S. Troian, in Organic Light-Emitting Materials and Devices III, edited by Z. H. Kafafi (SPIE, Bellingham, WA, 1999); Proc. SPIE 3797, 266 (1999). 